\title{
Educação Ambiental nos cursos de graduação em Engenharia Ambiental: ocorrência e formas de abordagem
}

\author{
Environmental Education in Environmental Engineering undergraduate \\ courses: occurrence and approach
}

\author{
Patrícia Ferreira de Oliveira ', Melchior José Tavares Júnior "
}

\section{RESUMO}

A Educação Ambiental é fundamental para lidar com os atuais problemas socioambientais da sociedade. De acordo com a legislação brasileira, a educação ambiental deve ser inserida em todos os níveis de ensino da educação formal. As universidades possuem o papel de produzir conhecimentos em acordo com as necessidades da sociedade, através da articulação entre ensino, pesquisa e extensão. O objetivo desta pesquisa foi avaliar a ocorrência e as formas de abordagem da Educação Ambiental nos cursos de Engenharia Ambiental do Brasil. Para tanto, foi desenvolvido uma pesquisa documental, analisando os Projetos Pedagógicos dos Cursos de uma amostra de cursos construída a partir de rankings tradicionais de avaliação no Brasil, incluindo o governamental. A Educação Ambiental está presente em 66,67\% da amostra que consideramos. Apenas quatro cursos possuem disciplinas obrigatórias, fato que preocupa, visto ser essa a única forma de garantir o acesso dos alunos ao conteúdo da Educação Ambiental. Projetos de pesquisa e de extensão deveriam estar mais presentes nos Projetos Pedagógicos dos Cursos. A forma predominante de ocorrência foi a disciplina optativa, o que deixa a possibilidade de que o Núcleo Docente Estruturante dos cursos, se for o caso, possa recomendar sua obrigatoriedade por ocasião da reformulação curricular.

Palavras-chave: Educação Ambiental; Engenharia Ambiental; Projetos Pedagógicos dos Cursos.

\section{ABSTRACT}

Environmental education is fundamental to deal with current society's problem. According to Brazilian law, environmental education must be included in all levels of education from formal education. Universities have the role to produce knowledge according to the needs of society through the articulation between teaching, research and extension. The objective of this research was to evaluate the occurrence and the ways of approach of EE in the Environmental Engineering courses in Brazil. Therefore, a documentary research was developed and, through it, we realize that the EE is present in $66.67 \%$ of the sample we consider. This is the case of UFU's Environmental Engineering course. Only four courses have obligatory subjects, and it concerned us, once this is the only way to ensure the access of students to EE content.

' Estudante de Engenharia Ambiental, Universidade Federal de Uberlândia. E-mail: patyferreira.2809@hotmail.com. ORCID: http://orcid.org/0000-0001-5758-5386.

" Professor efetivo no curso de Ciências Biológicas, Universidade Federal de Uberlândia/MG. E-mail: profmelk@hotmail.com. ORCID: https://orcid.org/0000-0002-5811-2828. 
Research and extension projects were little representative and should be more present in the PPCs of the courses. The predominant form of occurrence was the optional discipline, which makes us hopeful that the $\mathrm{NDE}$, if applicable, can recommend its obligation.

Keywords: Environmental Education; Environmental Engineering; Pedagogical Course Project.

\section{INTRODUÇÃO}

A Educação Ambiental (EA) é descrita como os processos por meio dos quais o indivíduo e a coletividade constroem valores sociais, conhecimentos, habilidades, atitudes e competências voltadas para a conservação do meio ambiente, bem de uso comum do povo, essencial à sadia qualidade de vida e a sua sustentabilidade (BRASIL, 1999).

O papel da EA vai além de discutir a relação homem-natureza, ela também se propõe a questionar o modelo social vigente, evitando a continuidade de propostas hegemônicas de reprodução social imposta pela construção de políticas que pouco, ou nada, representam uma maioria, apenas fortalecem as injustiças sociais e a desigualdade (ANDRADE, 2016). Para isso é necessário disseminar seus conhecimentos para a comunidade. Conforme Ubinski (2016, p.58), com quem concordamos:

Promover o conhecimento crítico é fundamental na Educação Ambiental. O conhecimento permite que os cidadãos possam lutar por seus direitos e se opor a injustiças sociais, envolvendo também aspectos relacionados ao ambiente em que vivem e os fatores que impactam na qualidade de vida das pessoas e de outros seres vivos. Assim, a Educação Ambiental precisa envolver os conteúdos científicos que possibilitem essa compreensão das problemáticas socioambientais.

É importante considerar que o processo de EA deve estar inserido na realidade e através de processos educativos, contribuir para sua transformação, desconstruindo e construindo tanto para o indivíduo quanto para a coletividade (SANT'ANA; MULLER; KALLÁS, 2019). Conforme os autores citados anteriormente, a educação assume posição de destaque para construir os fundamentos da sociedade sustentável, apresentando uma dupla função a essa transição societária: propiciar 
os processos de mudanças culturais em direção a instauração de uma ética ecológica e de mudanças sociais em direção ao empoderamento dos indivíduos, grupos e sociedades que se encontram em condições de vulnerabilidade face aos desafios da contemporaneidade.

A educação ambiental desde a década de 70 já vem sendo pensada, adotada em políticas de várias nações e fomentada com recursos para capacitação e produção de material didático e instrucional por organismos e programas da Organização das Nações Unidas (ONU). De acordo com Correa; Ashley (2018), ao verificarmos a interpretação das políticas em prol da educação ambiental em todos os níveis de ensino, não percebemos relevância e clareza para a sua inserção e muitas vezes é reduzida a sua interpretação a práticas cotidianas de cuidado com os resíduos domésticos e do ambiente da instituição de educação.

Os cursos de engenharia são estruturados abrangendo diversos conhecimentos e entendimentos técnicos, mas também pautados no contexto social, ambiental, econômico e político (ARAUJO, 2018). Diante da necessidade de profissionais capazes de desenvolver ações no meio antrópico com respeito ao meio natural foi proposto o curso de Engenharia Ambiental que abrange áreas biológicas, exatas e ciências sociais. O engenheiro ambiental deve ser responsável por solucionar questões ambientais, integrando - as com o desenvolvimento econômico e social.

De acordo com a portaria que institui o curso de Engenharia Ambiental no Brasil, a EA não é uma disciplina obrigatória (BRASIL, 1994). Entretanto, é fundamental para resolver problemas que serão objeto de trabalho do engenheiro ambiental.

Considerando a importância e urgência da $E A$, e que a mesma deve ser desenvolvida em todos os níveis da Educação formal, entendemos que o ensino superior tem um papel muito importante no estudo, formação e a promoção desse 
tema. Para tanto, a universidade não deve fugir à sua responsabilidade de investigar, compreender, sistematizar e divulgar o assunto. Neste estudo, o objetivo foi avaliar a ocorrência e as formas de abordagem da EA nos Projetos Pedagógicos dos cursos de Engenharia Ambiental do Brasil, visto que os profissionais formados nesse curso poderão ter que desenvolver projetos de EA em suas atividades.

\section{REFERENCIAL TEÓRICO}

\subsection{Legislação Brasileira}

A EA está presente no Brasil como um dos princípios da Lei $n^{\circ} 6.938$ que dispõe sobre a Política Nacional do Meio Ambiente:

Art. $2^{\circ}$ - A Política Nacional do Meio Ambiente tem por objetivo a preservação, melhoria e recuperação da qualidade ambiental propícia à vida, visando assegurar, no País, condições ao desenvolvimento sócio-econômico, aos interesses da segurança nacional e à proteção da dignidade da vida humana, atendidos os seguintes princípios: [...] X - Educação ambiental a todos os níveis de ensino, inclusive a educação da comunidade, objetivando capacitá-la para participação ativa na defesa do meio ambiente (BRASIL, 1981).

A Constituição Federal Brasileira de 1988 incumbe ao poder público promover a EA em todos os níveis de ensino e a conscientização pública para a preservação do meio ambiente. Em 1999, foi estabelecida a Lei $n^{\circ} 9.795$ que institui a Política Nacional de Educação Ambiental (PNEA). No artigo nono dessa lei fica definido que a EA deve ser desenvolvida em todos os níveis do ensino formal (BRASIL, 1999):

Art. $9^{\circ}$ Entende-se por educação ambiental na educação escolar a desenvolvida no âmbito dos currículos das instituições de ensino públicas e privadas, englobando: I - educação básica: a) educação infantil; b) ensino fundamental e c) ensino médio; II - educação superior; III - educação especial; IV - educação profissional; V educação de jovens e adultos.

A Resolução do Conselho Nacional de Educação n 02, de 15 de junho de 2012, estabeleceu as Diretrizes Curriculares Nacionais para a Educação Ambiental, passando a integrar o marco legal da EA no Brasil, apresentando-se como referência 
para a promoção da EA em todos os níveis e modalidades do ensino formal (BRASIL, 2012).

Conforme o artigo $10^{\circ}$ desse documento, as instituições de ensino superior devem utilizar dos princípios e objetivos da EA para promover sua gestão e suas ações de ensino, pesquisa e extensão. Além disso, fica estabelecido que a proposta curricular para inserção da EA é constitutiva dos Projetos Pedagógicos de Curso (PPC) e do Projeto Pedagógico (PP) constante do Plano de Desenvolvimento Institucional (PDI) das instituições de Educação Superior.

O artigo $16^{\circ}$ das Diretrizes Curriculares Nacionais para a Educação Ambiental aborda sobre a aplicação dos conhecimentos de EA nos currículos:

Art. 16. A inserção dos conhecimentos concernentes à Educação Ambiental nos currículos da Educação Básica e da Educação Superior pode ocorrer: I - Pela transversalidade, mediante temas relacionados com o meio ambiente e a sustentabilidade socioambiental; II - como conteúdo dos componentes já constantes do currículo; III - pela combinação de transversalidade e de tratamento nos componentes curriculares (BRASIL, 2012, p. 5).

Entretanto, esse tema também pode ocorrer como disciplina no ensino superior, conforme artigo $8^{\circ}$ (BRASIL, 2012, p. 3):

Art. $8^{\circ} \mathrm{A}$ Educação Ambiental, respeitando a autonomia da dinâmica escolar e acadêmica, deve ser desenvolvida como uma prática educativa integrada e interdisciplinar, contínua e permanente em todas as fases, etapas, níveis e modalidades, não devendo, como regra, ser implantada como disciplina ou componente curricular específico. Parágrafo único. Nos cursos, programas e projetos de graduação, pós-graduação e de extensão, e nas áreas e atividades voltadas para o aspecto metodológico da Educação Ambiental, é facultada a criação de componente curricular específico. Griffo nosso.

\subsection{Educação Ambiental na universidade}

No ano de 1975 ocorreu o Encontro Internacional de EA, em Belgrado, lugoslávia. Após o encontro, a EA foi introduzida pelo governo, nos setores educacionais brasileiros, entretanto era focada apenas nos aspectos biológicos do meio ambiente, desconsiderando os aspectos sociais, econômicos, políticos, 
culturais, etc. As questões político-ambiental se destacaram dentro das universidades brasileiras no ano de 1986, quando foi realizado o "I Seminário Nacional sobre Universidade e Meio Ambiente" em Brasília e nos anos de 1987 e 1988 foi promovido o "I Curso de Especialização em Educação Ambiental" na Universidade de Brasília.

Uma das formas de promover o interesse de um graduando relacionado ao conhecimento e prática da Educação Ambiental é levá-lo a reflexão e percepção dos conhecimentos já adquiridos, das necessidades de sua profissão e da importância de um aprendizado contínuo para assim tornar-se, não apenas um cidadão, mas também um profissional mais consciente de seu papel na promoção do desenvolvimento sustentável (ROMAO et al.,2020).

\subsection{O curso de Engenharia Ambiental da Universidade Federal de Uberlândia}

A Resolução n 05/2009 do Consun/UFU de 28/04/2009 dispõe sobre a criação do curso de Bacharelado em Engenharia Ambiental na Universidade Federal de Uberlândia, com início das atividades no primeiro semestre de 2010. A Portaria MEC/Seres no 867 de 09/11/2015 - DOU de 13/11/2015 estabelece o reconhecimento do curso. O curso é ofertado pelo Instituto de Ciências Agrárias (ICIAG) e tem como objetivo preparar o futuro profissional para desempenhar suas responsabilidades previstas na legislação federal e no Conselho Regional de Engenharia, Arquitetura e Agronomia (CREA) de cada Estado. Segundo o Projeto Pedagógico do Curso:

O fluxo curricular proposta pelo ICIAG encontra-se em concordância com a portaria n. ${ }^{\circ} 1693$ de 05 de dezembro de 1994 que dispõe sobre a criação do curso de Engenharia Ambiental e estabelece o currículo mínimo, possibilitando a formação de profissionais de atividades técnicas de atuação Engenheiro Ambiental, tais como: administração, gestão e ordenamento ambiental, monitoramento e mitigação de impactos ambientais, seus serviços afins e correlatos. Atividades como recuperação de áreas degradadas, gerenciamento de resíduos (urbanos, agrícolas e industriais), avaliação de impactos ambientais, medidas mitigadoras de controle de poluição, educação ambiental e planejamento e implantação de sistemas de gerenciamento ambiental (SGA), igualmente 
mostram-se como pertinentes ao campo de trabalho do Engenheiro Ambiental. Essas atividades serão atingidas em sua plenitude por meio de disciplinas propostas no fluxo curricular, elaborado pela comissão (ICIAG,2019, p. 14).

A EA se apresenta como disciplina optativa do curso, com carga horária total de 60 horas. Atualmente, essa disciplina está sendo regularmente oferecida todos os semestres. Entretanto, são ofertadas 15 vagas por semestre, cinco para o turno matutino e 10 para o turno noturno, além da possibilidade das vagas remanescentes da disciplina ofertada para o curso de Biologia. Esse número reduzido de vagas deve-se ao fato da disciplina ser oferecida no mesmo horário da disciplina oferecida para o curso de Biologia.

Em 2020, o atual curso de Engenharia Ambiental passará a se chamar Engenharia Ambiental e Sanitária, resultado da reformulação curricular. Conforme seu PPC (ICIAG,2019), a mudança permitirá ao futuro profissional de engenharia ambiental e sanitária atuar em áreas como projeto e acompanhamento de obras de tratamento de água e esgoto, aterros sanitários, drenagem urbana, barragens, que não são permitidas para o profissional formado em engenharia ambiental. A disciplina de EA continuará como optativa, com carga horária de 60 horas.

\section{METODOLOGIA}

Para responder a pergunta de pesquisa, entende-se que o PPC é o lugar adequado para investigação. O PCC é um instrumento que define os princípios filosóficos, políticos, pedagógicos, administrativos e técnicos a serem adotados na condução do processo de ensino aprendizagem da Graduação, contemplando os objetivos gerais do curso, matriz curricular, etc. (UFES, 2016). Assim, essa pesquisa configura-se como uma pesquisa documental.

Para o levantamento de dados, foram escolhidas três fontes: (1) Ranking Universitário Folha (RUF); (2) Guia do Estudante e (3) Instituto Nacional de Estudos e Pesquisas Educacionais Anísio Teixeira (Inep)/ Ministério da Educação (MEC). 


\subsection{Ranking Universitário Folha (RUF)}

O RUF é uma avaliação anual do ensino superior do Brasil feita pela folha desde 2012. A avaliação é feita anualmente com base em dados nacionais e internacionais e em duas pesquisas de opinião do Datafolha em dois aspectos (ensino e mercado).

\section{Tabela 1 - Ranking Universidade Folha 2018}

\begin{tabular}{lcc}
\hline Posição no país & Nome da Instituição & UF \\
\hline $1^{\circ}$ & Universidade de São Paulo (USP) & SP \\
$2^{\circ}$ & Universidade Federal do Rio de Janeiro (UFR) & RJ \\
$3^{\circ}$ & Universidade Federal de Minas Gerais (UFMG) & MG \\
$4^{\circ}$ & Universidade Federal do Rio Grande do Sul (UFRGS) & RS \\
$5^{\circ}$ & Universidade Federal de Santa Catarina (UFSC) & SC \\
$6^{\circ}$ & Universidade Estadual de Campinas (UNICAMP) & SP \\
$7^{\circ}$ & Universidade Federal do Paraná (UFPR) & PR \\
$8^{\circ}$ & Universidade Federal de São Carlos (UFSCAR) & SP \\
$9^{\circ}$ & Universidade Estadual Paulista Júlio de Mesquita Filho & SP \\
$10^{\circ}$ & Universidade Federal de ViçOsa (UFV) & MG \\
$11^{\circ}$ & Universidade Federal de Santa Maria (UFSM) & RS \\
$12^{\circ}$ & Universidade de Brasília (UNB) & DF \\
$13^{\circ}$ & Universidade Federal do Ceará (UFC) & CE \\
$14^{\circ}$ & Universidade da Região de Joinville (UNIVILLE) & SC \\
$15^{\circ}$ & Universidade Federal do Mato Grosso (UFMT) & MT \\
\hline
\end{tabular}

Fonte: Ranking Universitário Folha, 2018. Acesso em 2019. 


\subsection{Guia do estudante}

A avaliação dos cursos superiores do Guia do Estudante conta com mais de 25 edições realizadas, sendo iniciada na década de 1990. A avaliação é uma pesquisa de opinião feita, basicamente, com professores e coordenadores de curso. Eles emitem conceitos que permitem classificar os cursos em bom (três estrelas), muito bons (quatro estrelas) e excelentes (cinco estrelas).

Tabela 2 - Avaliação guia do estudante 2017

\begin{tabular}{lcc}
\hline Instituição & Cidade UF & Classificação \\
\hline UFC & Fortaleza CE & $\star \star \star \star \star$ \\
UNB & Brasília DF & $\star \star \star \star \star$ \\
UFLA & Lavras MG & $\star \star \star \star \star$ \\
UFOP & Ouro preto MG & $\star \star \star \star \star$ \\
UFV & Viçosa MG & $\star \star \star \star \star$ \\
Unesp & Rio Claro SP & $\star \star \star \star \star$ \\
USP & São Paulo SP & $\star \star \star \star \star$ \\
Unesp & Sorocaba SP & $\star \star \star \star \star$ \\
UFSC & Florianópolis SC & $\star \star \star \star \star$ \\
UFRA & Belém PA & $\star \star \star \star$ \\
UFT & Palmas TO & $\star \star \star \star$ \\
UFAL & Maceió AL & $\star \star \star \star$ \\
UFBA & Salvador BA & $\star \star \star \star$ \\
UEPB & Campina Grande PB & $\star \star \star \star$ \\
UFPB & João Pessoa PB & $\star \star \star \star$
\end{tabular}

Fonte: Guia do Estudante, 2017. Acesso em 2019. 


\subsection{Inep/MEC}

O Inep realiza uma avaliação dos cursos de graduação através de um indicador de qualidade conhecido como Conceito Preliminar de Curso (CPC), esta avaliação teve início no ano de 2007. Os insumos utilizados nos cálculos são: desempenho dos estudantes no Enade valor agregado pelo curso ao desenvolvimento dos estudantes concluintes (IDD), perfil do corpo docente e percepção discente sobre as condições do processo formativo. Foram selecionadas as instituições com CPC 5 e 4.

Tabela 3 - Avaliação realizada pelo Inep através do CPC: Engenharia Ambiental, 2018

\begin{tabular}{lcc}
\hline Instituição & Cidade UF & CPC \\
\hline Unesp & São José dos Campos SP & 5 \\
UFRGS & Porto Alegre RS & 5 \\
UFERSA & Pau dos Ferros RN & 5 \\
UNIRITTER & Porto Alegre RS & 5 \\
FAJ & Jaguariúna SP & 5 \\
UNICAPE & Cariacica ES & 5 \\
Facear & Curitiba PR & 5 \\
UFMT & Rondonópolis MT & 4 \\
UFMT & Cuiabá MT & 4 \\
UFMT & Sinop MT & 4 \\
UNB & Brasília DF & 4 \\
UFS & São Cristóvão SE & 4 \\
UFOP & Ouro Preto MG & 4 \\
UFV & Viçosa MG & 4 \\
PUCPR & Curitiba PR & 4 \\
UCS & Caxias do Sul RS & 4 \\
UFU & Uberlândia MG & 4 \\
UPF & Passo Fundo RS & 4 \\
UDESC & Lages SC & 4 \\
UNESP & Presidente Prudente SP & 4 \\
\hline
\end{tabular}




\begin{tabular}{|c|c|c|}
\hline Instituição & Cidade UF & CPC \\
\hline UNESP & Rio Claro SP & 4 \\
\hline UNIVILLE & Joinville SC & 4 \\
\hline UNIVALI & Itajaí SC & 4 \\
\hline USC & Bauru SP & 4 \\
\hline UNESA & Nova Friburgo RJ & 4 \\
\hline UVA & Cabo Frio RJ & 4 \\
\hline CEUNIH & Belo Horizonte MG & 4 \\
\hline UNISANTOS & Santos SP & 4 \\
\hline UNOESTE & Presidente Prudente SP & 4 \\
\hline UNIVAP & São José dos Campos SP & 4 \\
\hline UNISC & Santa Cruz do Sul RS & 4 \\
\hline UEPB & Campina Grande & 4 \\
\hline UFRN & Natal RN & 4 \\
\hline UFF & Niterói RJ & 4 \\
\hline UFES & Vitória ES & 4 \\
\hline UFMG & Belo Horizonte MG & 4 \\
\hline UFJF & Juiz de Fora MG & 4 \\
\hline UFAL & Maceió AL & 4 \\
\hline UFBA & Salvador BA & 4 \\
\hline UFPB & João Pessoa PB & 4 \\
\hline UFC & Fortaleza CE & 4 \\
\hline UFSM & Santa Maria RS & 4 \\
\hline UFSM & Frederico Westphalen RS & 4 \\
\hline UFSC & Florianópolis SC & 4 \\
\hline UFRJ & Rio de Janeiro RJ & 4 \\
\hline UTFPR & Londrina PR & 4 \\
\hline UTFPR & Medianeira PR & 4 \\
\hline UTFPR & Francisco Beltrão PR & 4 \\
\hline UFRA & Belém PA & 4 \\
\hline UFLA & Lavras MG & 4 \\
\hline CEFET/MG & Belo Horizonte MG & 4 \\
\hline
\end{tabular}




\begin{tabular}{lcc}
\hline Instituição & Cidade UF & CPC \\
\hline UFTM & Uberaba MG & 4 \\
UFPEL & Pelotas RS & 4 \\
USF & Campinas SP & 4 \\
UNIR & Ji-Paraná RO & 4 \\
FAMEC & Camaçari BA & 4 \\
FDV & Viçosa MG & 4 \\
IFCE & Quixadá CE & 4 \\
IFES & Vitória ES & 4 \\
IFG & Goiânia GO & 4 \\
UFABC & Santo André & 4 \\
UFFS & Cerro Largo RS & 4 \\
UFFS & Chapecó SC & 4 \\
UFOB & Barreiras BA & 4 \\
\hline
\end{tabular}

Fonte: Portal Inep, 2018. Acesso em 2019.

De modo a obter as instituições a serem analisadas nesta pesquisa, buscou-se a ocorrência da instituição/curso em pelos menos duas das três fontes consideradas, através da comparação de tabelas. Os resultados estão representados na tabela 4. Utilizando a palavra-chave: Educação Ambiental, pesquisou-se no Projeto Pedagógico das instituições selecionadas se o tema é abordado e, em caso positivo, verificando se o mesmo encontra-se como disciplina obrigatória ou disciplina optativa, em projetos de pesquisa e extensão, ou ainda nas Empresas Júnior e no Programa de Educação Tutorial (PET) do curso, ou ainda em outra forma, conforme recomendação das diretrizes nacionais para a EA. O item a seguir, apresenta os resultados encontrados, com base nos referenciais adotados. 


\section{RESULTADOS E DISCUSSÕES}

Optou-se por inserir neste item o primeiro resultado que foi verificar a ocorrência de cada uma das instituições em pelo menos duas das três tabelas anteriores. Foram selecionadas 21 instituições, as quais estão listadas na tabela 4:

\section{Tabela 4 - Instituições selecionadas para análise do PPC}

\begin{tabular}{lccc}
\hline $\mathbf{N}^{\circ}$ & Instituição & Cidade & UF \\
\hline 1 & UFRA & Belém & PA \\
2 & UFMG & Belo & MG \\
3 & UNB & Brasília & DF \\
4 & UEPB & Campina & PB \\
5 & UFMT & Cuiabá & MT \\
6 & UFSC & Florianópolis & SC \\
7 & UFC & Fortaleza & CE \\
8 & UFPB & João Pessoa & PB \\
9 & UNIVILLE & Joinville & SC \\
10 & UFLA & Lavras & MG \\
11 & UFAL & Maceió & AL \\
12 & UFOP & Ouro Preto & MG \\
13 & UFT & Palmas & TO \\
14 & UFRGS & Porto Alegre & RS \\
15 & UNESP & Rio Claro & SP \\
16 & UFRJ & Rio de Janeiro & RJ \\
17 & UFBA & Salvador & BA \\
18 & UFSM & Santa Maria & RS \\
19 & USP & São Paulo & SP \\
20 & UNESP & Sorocaba & SP \\
21 & UFV & Viçosa & MG \\
\hline
\end{tabular}

Fonte: Elaborado pelos autores. 
Os PPCs foram consultados através dos sites das referidas instituições, cinco delas não apresentaram o PPC on-line. Nesse caso, considerou-se a matriz curricular dos mesmos. Nos casos em que o PPC não continha a matriz curricular, considerouse os dois arquivos separados: tanto o próprio PPC como sua matriz de disciplinas.

Das 21 instituições, 12 possuem o curso de Engenharia Ambiental (UFMG, UNB, UFC, UFPB, UFOP, UFT, UFRGS, UNESP Rio Claro, UFRJ, USP, UNESP Sorocaba e UFV), oito possuem o curso de Engenharia Ambiental e Sanitária (UEPB, UFMT, UFSC, UNIVILLE, UFLA, UFAL, UFBA e UFSM) e a UFRA possui o curso de Engenharia Ambiental e Energia Renováveis.

Conforme previsto em nossa metodologia, utilizamos a palavra-chave Educação Ambiental e obtivemos os seguintes resultados:

\section{Gráfico 1 - Resultado da consulta aos PPC's e matrizes curriculares}

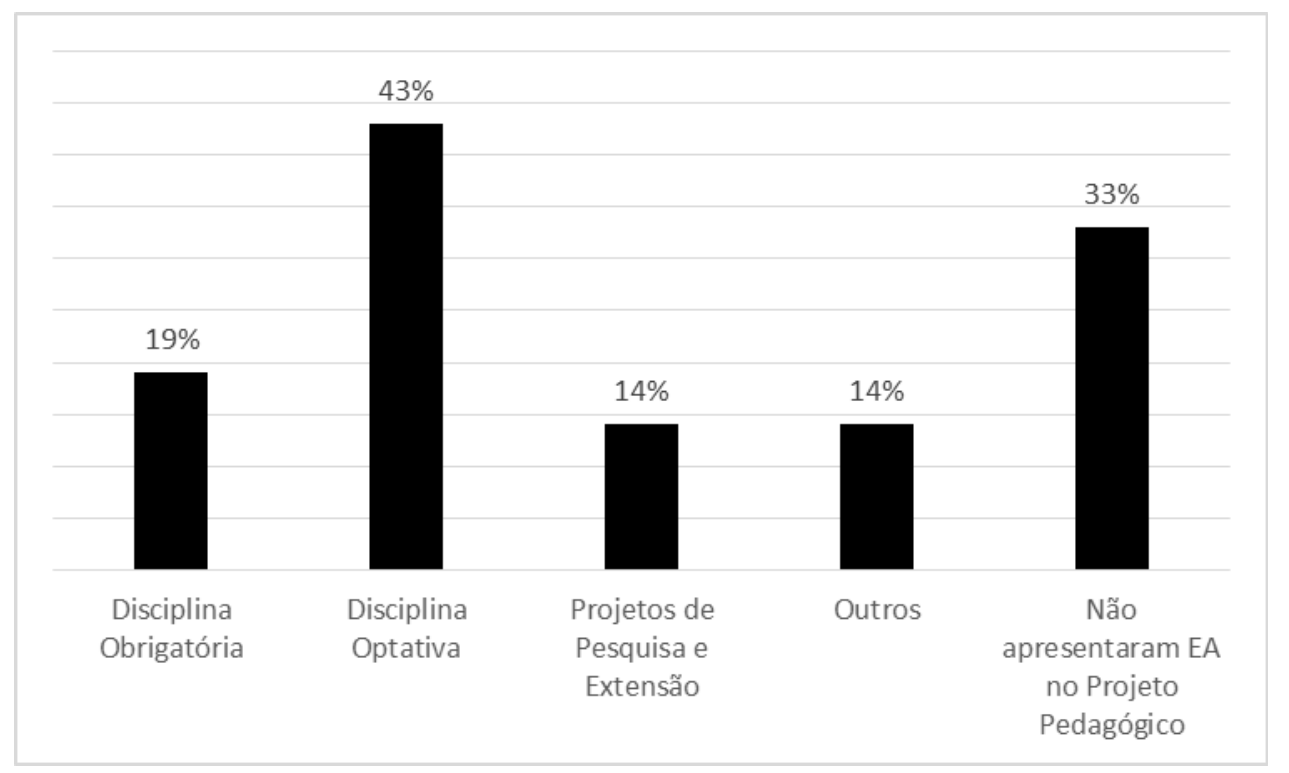

Fonte: Elaborado pelos autores.

Recuperando o objetivo de pesquisa, observa-se que, por esta metodologia utilizada, a EA está presente em $66,67 \%$ dos cursos ou seja, em aproximadamente $2 / 3$ da amostra considerada. À primeira vista, esse resultado é positivo, pois contempla um assunto importante para o engenheiro ambiental, inclusive previsto 
em lei (BRASIL, 1999; 2012). Os dados também evidenciam que essa ocorrência se dá de várias formas, embora predomine a forma disciplinar.

A EA ocorre como disciplina obrigatória em quatro instituições: UFRA, UFPB, UFT e UFRGS, menos de $20 \%$ do total da amostra. Esse resultado não é satisfatório. A preocupação com o número reduzido de cursos com disciplinas obrigatórias está no fato de que é apenas nessa condição de obrigatoriedade que os alunos terão garantido o acesso ao conteúdo da EA. A carga horária média dessas disciplinas são 50 horas, bastante próximo ao padrão da maioria das disciplinas acadêmicas, que é de 60 horas.

Este tema ocorre como disciplina optativa em nove instituições: UNB, UEPB, UFMT, UFSC, UFC, UFLA, UFOP, UFRJ e UFV, representando 43\%. Esse resultado é bastante satisfatório. Essa inserção geralmente é resultado do interesse de um ou alguns professores e alunos. Embora a disciplina optativa não garanta acesso ao conteúdo, há a possibilidade de mudar o status da disciplina, tornando-a uma disciplina obrigatória. Para tanto, é necessário a investigação do Núcleo Docente Estruturante (NDE) junto aos alunos e, se for o caso, a recomendação da mudança de status à coordenação do curso, especialmente por ocasião de reformulação curricular. A carga horária média nas instituições é de 49 horas, novamente próximo ao padrão da maioria das disciplinas acadêmicas, que é de 60 horas.

As instituições que desenvolvem projetos de pesquisa e extensão em EA são UFRA, UFSC e UNIVILLE. A primeira instituição apresenta o Núcleo em Educação Ambiental (NEA), um laboratório desenvolvido para realizar atividades de ensino, pesquisa e extensão nas seguintes áreas: EA; capacitação de gestores e educadores ambientais; capacitação das comunidades envolvidas no processo de gestão; produção e divulgação de materiais educativos. A UFSC conta com o Núcleo de Educação Ambiental (NEAMB), uma iniciativa de estudantes do curso de Engenharia Sanitária e Ambiental, o grupo é composto por estudantes envolvidos em projetos 
interdisciplinares de extensão universitária e desenvolve pesquisas com a aplicação de tecnologias sociais, através do suporte técnico e do conhecimento gerado na universidade. Com objetivo de inserir e trabalhar as questões socioambientais em todos os centros da UFSC e nas comunidades de atuação dos projetos voltados para a sustentabilidade em escolas, unidades de conservação, bacias hidrográficas e municípios. Na UNIVILLE os projetos de pesquisa e extensão estão associados ao Programa de Assessoria Técnico-Científica ao Comitê de Gerenciamento da Bacia Hidrográfica do Rio Cubatão Norte e Cachoeira (CCJ) e as pesquisas desenvolvidas relacionam a EA com o uso e gerenciamento dos recursos hídricos.

Os resultados mostram que a ocorrência de pesquisas em EA nos cursos de Engenharia Ambiental é reduzida, contrariando as recomendações desde a conferência de Tbilisi até as diretrizes nacionais para que haja ênfase na pesquisa em EA (BRASIL, 2012) e evidenciam a baixa aplicação da EA em projetos de extensão dentro dos espaços universitários. A extensão universitária é a principal forma de socializar o conhecimento entre estudantes e sociedade.

Apesar da baixa carga horária semestral para a disciplina obrigatória na UFRGS, o curso oferece a opção de estágio curricular em EA como disciplina alternativa composta por 110 horas e com o objetivo principal de capacitar o futuro profissional a atuar como mediador das demandas sociais e da necessidade de controle e/ou recuperação ambientais. O Estágio em EA abrange aspectos relacionados à educação de comunidades para preservação e controle ambiental do seu espaço físico, até aspectos relacionados a reeducação de comunidades que experimentaram, ou estão experimentando mudanças ambientais significativas em seu meio. A UEPB apresenta em seu PPC a PNEA como base legal para a construção e caracterização do curso. Dentre todos os PPC's, foi a única instituição que inseriu esse elemento. Na UNIVILLE, além dos projetos de pesquisa e extensão, a EA 
também é abordada de forma transversal por meio de outras disciplinas, como Planejamento e Gestão Ambiental.

\section{CONCLUSÕES}

Os resultados obtidos demonstram que a EA está inserida com alta frequência nos Projetos Pedagógicos dos Cursos de Engenharia Ambiental selecionados. Entretanto devido a sua necessidade, o tema deveria ser abordado de forma mais detalhada para despertar o interesse dos futuros engenheiros ambientais e promover na prática conhecimentos para que estes sejam usados na resolução de problemas da sociedade.

\section{REFERÊNCIAS}

ANDRADE, R. J. Educação Ambiental da teoria à prática: ação interventiva no Instituto Federal da Bahia - IFBA no município de Valença-BA. 2016. 107 f. Dissertação (Mestrado) - Curso de Engenharia Agronômica, Universidade Federal do Recôncavo da Bahia, Cruz das Almas, 2016.

ARAUJO, E. (2018). Sustentabilidade e Engenharia Ambiental desafios na formação do profissional cidadão. Revista Dissertar,1(30). https://doi.org/10.24119/16760867ed114244

BRASIL. Ministério da Educação. Conselho Nacional de Educação. Resolução n 2, de 15 de junho de 2012. Estabelece as Diretrizes Curriculares Nacionais para a Educação Ambiental. Diário Oficial do Poder Executivo, Brasília, 18 jun. 2012.

. Lei $n^{\circ}$ 9795, de 27 de abril de 1999. Dispõe sobre a educação ambiental, institui a Política Nacional de Educação Ambiental e dá outras providências. Brasília, 27 de abril de 1999.

Ministério da Educação e Desporto. (1994). Institui o curso de Engenharia Ambiental no Brasil. Portaria n. 1.693. Brasília, 05 de dezembro de 1994.

. Lei $n^{\circ}$ 6938, de 31 de agosto de 1981. Dispõe sobre a Política Nacional do Meio Ambiente, seus fins e mecanismos de formulação e aplicação, e dá outras providências... Brasília, 31 de agosto de 1981. 
CORRÊA, M. M.; ASHLEY, P. A. Desenvolvimento Sustentável, Sustentabilidade, Educação Ambiental e Educação para o Desenvolvimento Sustentável: Reflexões para ensino de graduação. REMEA - Revista Eletrônica do Mestrado em Educação Ambiental, [S.I.], v. 35, n. 1, p. 92-111, maio 2018. ISSN 1517-1256. Disponível em: https://periodicos.furg.br/remea/article/view/7417. Acesso em: 20 mar. 2020. doi: https://doi.org/10.14295/remea.v35i1.7417.

ICIAG (Minas Gerais). Universidade Federal de Uberlândia. Projeto Pedagógico do Curso de Engenharia Ambiental. Disponível em: http://www.iciag.ufu.br/sites/iciag.ufu.br/files/media/documento/projeto_pedagogico.atu alizado_com_normas_de_atividades_complementares_em_anexo_atualizado.pdf. Acesso em: 26 set. 2019.

RIBEIRO, E. F. DE S.; FERREIRA, M. S. Inserção da Educação Ambiental nos projetos pedagógicos dos cursos de direito: uma análise na região metropolitana do Rio de Janeiro. Revista Brasileira de Educação Ambiental, v. 14, n. 1, p. 316-338, 30 mar. 2019. Disponível em: https://periodicos.unifesp.br/index.php/revbea/article/view/2719. Acesso em: 17 nov. 2019.

ROMAO, E. L. et al (2020). Percepção ambiental de alunos de graduação em engenharia sobre a importância da Educação Ambiental. Revista Brasileira De Educação Ambiental (RevBEA),15(1), 194-208. https://doi.org/10.34024/revbea.2020. v15.10060.

SANT'ANA, L. C. F.; MULLER, E.; KALLÁS, F. Projetos de educação ambiental em universidades: uma reflexão necessária e urgente. Brazilian Journal of Animal And Environmental Research, Curitiba, v. 2, n. 3, p.917-927, maio 2019.

UBINSK, J. A. S. Análise de atividades de complementação curricular na área de Educação Ambiental e suas contribuições à Alfabetização Científica. 2016. 207 f. Dissertação (Mestrado Educação) - Universidade Estadual do Oeste do Paraná, Cascavel, 2016.

UFES. Diretrizes para elaboração de Projeto Pedagógico de curso - PPC (Versão Preliminar). $2016 . \quad$ Disponível em: http://prograd.ufes.br/sites/prograd.ufes.br/files/field/anexo/diretrizes_ppc_-_04-102016.pdf. Acesso em: 02 jun. 2019. 